\title{
Psychiatric Discharge Summaries in Mental Handicap Settings
}

\author{
George Ikkos, Senior Registrar in Psychiatry, University College Hospital, London WC1
}

Selective abstraction of information and good communication are essential in the practice of psychiatry. A good psychiatric discharge summary performs both these functions and can therefore be a significant aid in patient management. The need for a good psychiatric discharge summary is no less necessary in the psychiatry of mental handicap than in general adult psychiatry. However, the psychiatry of mental handicap does differ in some respects from that of general adult psychiatry. This difference therefore should be reflected in the psychiatric discharge summary in mental handicap settings if the summary is to represent accurately the clinical process. This fact was recognised by the Medical Executive Committee of Leavesden Hospital and it was therefore decided to provide specific guidelines on the writing of discharge summaries in a mental handicap in-patient setting to junior trainees in that hospital. Below is a document which I prepared at the request of the Medical Executive Committee, and which has now been officially accepted by them. It is hoped that its publication will be of interest to readers working in other mental handicap hospitals.

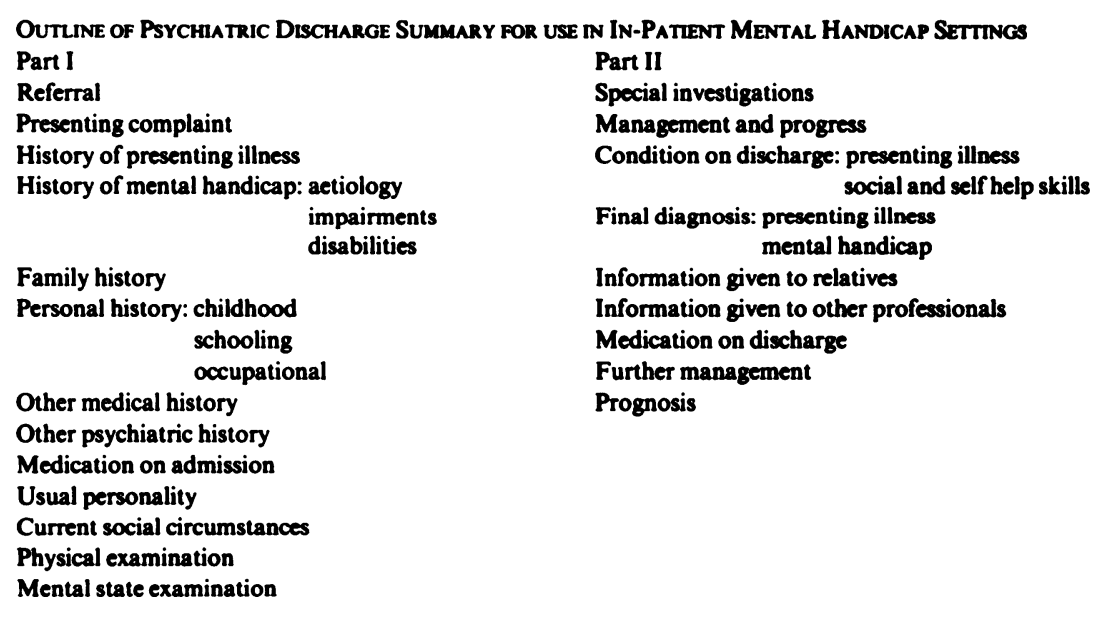

\begin{abstract}
Notes on Writing Psychiatric Discharge Summaries in In-Patient Mental Handicap Settings (Please refer to Outline)
\end{abstract}

Psychiatric Discharge Summaries are important documents, which are recognised as such by psychiatrists ${ }^{1}$ and valued highly by GPs. The quality of your involvement with the patient will probably be reflected in the Summary. A brief but comprehensive Summary usually indicates concerned involvement by a doctor who has the capacity to think intelligently about his patient. The writing of the Summary itself should be used as a learning opportunity and it is assumed that you are familiar with writing general psychiatric Summaries in the discussion below.

The overall format of the Summary is that generally accepted in psychiatry ${ }^{3}$; but modified according to recent research findings. ${ }^{2}$ You are strongly advised to consult these documents. Part I of the Summary should be completed within two weeks of admission to hospital. Part II should be completed within one week of discharge from 
hospital. The completed Summary should not occupy more than two sides of an A4 sheet of paper. To achieve this, while using all the readings of the outline, the following tactics should be used.

1. The title of each section should be in capital letters and underlined e.g. PRESENTING COMPLAINT

2. Double spacing should be used only when moving from one section to the next e.g. PRESENTING COMPLAINT to HISTORY OF MENTAL HANDICAP

3. Each section should be like a paragraph typed with singled spacing and continuing directly from the title of the section e.g. FAMily HISTORY: Father, 58yrs, shopkeeper, caring and considerate, heart attack three years ago, Mother, 55yrs...

4. Use a telegraphic style when writing (see example in point 3 above)

5. Ask your secretary to type on both sides of the paper.

Patients are not referred to psychiatrists because they are handicapped. It is important, therefore, to distinguish clearly between the history of the presenting illness (e.g. epilepsy or psychosis) in particular from that of mental handicap in general. In completing the section on 'HISTORY OF MENTAL HANDICAP' the following WHO definitions ${ }^{4}$ will be helpful:

MPAIRMENT: 'Any loss or abnormality or psychological, physiological or anatomical structure'. The important thing is that impairments are 'neutral or objective descriptions of the site, nature and severity of loss of structure or functional capacity'. This loss may be anatomical (e.g. microcephaly), biochemical (e.g. phenylketonuria), or mental (e.g. low IQ) in nature.

DISABILITY: 'Any restriction or lack (resulting from an impairment) of ability to perform an activity or to perform it within the range considered normal for a human being'. Disabilities may be physical (e.g. partial blindness) or psychological (e.g. learning disabilities).

Remember always to include epilepsy in the section on the HISTORY OF MENTAL HANDICAP when it is present and it is not the presenting complaint.

The sections on 'OTHER MEDICAL hISTORY' and 'OTHER PSYCHIATRIC HISTORY' should include conditions that cannot be aetiologically related to mental handicap. Regarding personality, the term 'USUAL' is preferred to 'PREMORBID' because morbidity in these patients often dates back to earliest childhood. The term 'Usunl' should be taken to refer to the personality before the onset of the presenting illness.

The results of all investigations (including EEG, IQ etc) during the current admission should be included under 'SPECIAL INVESTIGATIONS'. In describing 'CONDITION ON DISCHARGE' describe the patients' condition in relation to the presenting complaint/illness and give a clear idea of their social and self-help skills. When coming to a final diagnosis regarding mental handicap state the aetiology if known, and the degree of handicap (borderline/mild/moderate/ severe). It is appropriate to refer here to major disabilities like blindness and epilepsy when present. When referring to epilepsy make clear what type it is (e.g. complex partial seizures, major tonic clonic seizures etc.) Research indicates that 'INPORMATION GIVEN TO RELATIVES' is particularly valued by GPs." The section on 'INFORMATION GIVEN to OTHER PROFESSIONALS' is included here because many mentally handicapped live in hostels, group homes, or have involvement by multiple professionals (social workers, community nurses, etc). Both sections should be brief (i.e. no more than one short sentence per relative or professional referred to).

When giving information about 'FURTHER MANAGEMENT' be precise (i.e. give names of professionals involved, location of involvement with patient and, if possible, dates). Also make explicit who is responsible for further management (i.e. GP, consultant psychiatrist etc). Remember to include all relevant information in the Summary. Remember also to write a short Summary. A long Summary is a contradiction in terms and raises serious questions about your ability to selectively abstract clinically relevant information. Finally, remember that a good Summary can be a reliable reference source, and thus a valuable tool in the future management of the patient.

\section{ReFerences}

${ }^{1}$ GeLDER, M., GATH, D. \& MAYOU, R. (1983) Oxford Textbook of Psychiatry. Oxford: Oxford University Press, p. 59.

${ }^{2}$ OrReLL, M. W. \& Greenber G, M. (1986) What makes psychiatric summaries useful to general practitioners. Bulletin of the Royal College of Psychiatrists, 10, 107-109.

3Department of Psychatry Teachng Comadter-The InstitUTE Of Psychatrey (1973) Notes on Eliciting and Recording Clinical Information. Oxford: Oxford University Press, p. 12.

${ }^{4}$ RusseuL, O. (1985) Mental Handicap. Edinburgh: Churchill Livingstone, p. 6.

\section{Correction}

Practical Management: Impotence. By Raymond E. Goodman (Bulletin, April 1987, 11, 125). The second reference should have read:

Melman, A. (1978) Development of contemporary surgical management for erectile impotence. Sexwality and Disability. $1,4,272-281$.
References 3 and 4 also refer to this Journal.

Dr Goodman's present address is: Psychosexual Clinic, Lancastrian Unit, Hope Hospital, Eccles Old Road, Salford M6 8HD. 